CERN-TH.6685/92

UPR-0526T

\title{
NATURAL SUPERGRAVITY INFLATION
}

\author{
Gabriel Lopes Cardoso \\ and \\ Burt A. Ovrutf \\ Theory Division, CERN, CH-1211 Geneva 23, Switzerland \\ and \\ Department of Physics, University of Pennsylvania, \\ Philadelphia, PA 19104-6396, USA
}

\begin{abstract}
We show that a single uncharged chiral superfield, canonically coupled to $N=1$ supergravity with vanishing superpotential, naturally drives inflation in the early universe for a class of simple Kahler potentials. Inflation occurs due to the one-loop generation of a Kahler anomaly proportional to $\mathcal{R}^{2}$. The coefficient of this $\mathcal{R}^{2}$ term is of the correct magnitude to describe all aspects of an inflationary cosmology, including sufficient amplitude perturbations and reheating. Higher order terms proportional to $\mathcal{R}^{n}$ for $n \geq 3$ are naturally suppressed relative to the $\mathcal{R}^{2}$ term and, hence, do not destabilize the theory.
\end{abstract}

CERN-TH.6685/92

UPR-0526T

October 1992

\footnotetext{
${ }^{1}$ Work supported in part by the Department of Energy under Contract No. DOE-AC02-76-ERO3071.
} 
It has been known for some time that one can achieve inflationary growth in the early universe by modifying the usual Einstein gravity Lagrangian[1]. This is accomplished by adding the term $\epsilon \mathcal{R}^{2}$, where $\mathcal{R}$ is the scalar curvature and $\epsilon$ is a dimensionless constant. Furthermore, it was shown in [2] that all the other physical requirements for an inflationary cosmology, such as sufficient amplitude perturbations and baryogenisis, are satisfied if parameter $\epsilon$ lies in the range $10^{11} \lesssim \epsilon \lesssim 7 \times 10^{15}$. This scenario, although potentially a very simple theory of inflation, suffers from two obvious deficiencies. First, why should one introduce an $\mathcal{R}^{2}$ term and, second and more seriously, why is $\epsilon$ so large when one would expect it to be of order one? These deficiencies were not addressed in [1, 2]. Furthermore, if one can introduce $\epsilon \mathcal{R}^{2}$, then what about terms like $\epsilon^{(n)} \mathcal{R}^{n}$ where $n \geq 3$ ? Shouldn't these terms also be added and if not, why not? A careful study of the effect of such terms on inflation was presented in [3, 田. It was shown that higher $\mathcal{R}^{n}$ terms tend to destabilize the $\mathcal{R}^{2}$ cosmological scenario if the $\epsilon^{(n)}$ coefficients are sufficiently large. It would appear then, that these deficiencies would have to be overcome before the $\epsilon \mathcal{R}^{2}$ cosmology can be taken seriously. In this paper, we present a simple particle physics theory in which all of these deficiencies are resolved in a natural manner.

Recently, it has become clear that the classical Kahler symmetry of four-dimensional, $N=1$ supergravity-matter theories is, in general, broken by an anomaly at the one-loop level. Part of this anomaly arises from the coupling of the composite Kahler connection to two gauge fields via a triangle graph. This process leads to non-holomorphic corrections to gauge couplings and threshold effects [5, 6]. More importantly, from the point of view of this paper, is another contribution to the Kahler anomaly which arises from the coupling of the Kahler connection to two gravitons via a triangle graph. This process was first introduced in [5]. For simplicity, this anomaly was computed in [5] with the gravitons on-shell, and was found to be proportional to $\left(C_{m n p q}\right)^{2}$ and $\mathcal{R}_{\text {mnpq }} \tilde{\mathcal{R}}^{\text {mnqp}}$. However, in this paper, we will show that when this result is extended off-shell, a non-topological term proportional to $\mathcal{R}^{2}$ must arise due to the constraints of supersymmetry. That is, terms proportional to the square of the scalar curvature arise naturally in $N=1$ supergravity theories through the one-loop Kahler anomaly. Furthermore, the coefficient of $\mathcal{R}^{2}$ is not a constant but rather is found to be a specific function of scalar fields, the Kahler potential. We will show in this paper that, for 
a class of simple Kahler potentials, this coefficient can be naturally very large, easily in the range required in [2]. Finally, we find that all radiative corrections involving $\mathcal{R}^{n}$ for $n \geq 3$ are, since they are Kahler invariant, strongly suppressed relative to the anomalous $\mathcal{R}^{2}$ term. Hence, these terms do not destabilize the $\mathcal{R}^{2}$ cosmology. Thus a class of simple four-dimensional, $N=1$ supergravity theories generically exhibit $\epsilon \mathcal{R}^{2}$ type cosmological behaviour, while naturally resolving all the associated deficiencies. The simplest such theory, which we discuss in this paper, consists of a single chiral superfield with vanishing superpotential coupled to canonically normalized Einstein supergravity. Remarkably, the radiative corrections to this theory naturally lead to $\epsilon \mathcal{R}^{2}$ inflationary cosmology without any of the deficiencies.

We begin by presenting a way of generating $\mathcal{R}^{2}$ terms with field dependent coefficients in the effective action for matter coupled to supergravity. These $\mathcal{R}^{2}$ terms arise from one-loop triangle graphs that are anomalous under Kahler transformations. First, let us recall a few fundamental facts about four-dimensional chiral matter coupled to supergravity. The most general tree-level Lagrangian describing the coupling of chiral matter superfields $\Phi^{i}$ to supergravity is determined by three functions [7]; the Kahler potential $K\left(\Phi^{i}, \Phi^{i \dagger}\right)$, the holomorphic superpotential $W\left(\Phi^{i}\right)$ and the holomorphic gauge coupling function $f_{a b}\left(\Phi^{i}\right)$. In this paper, only the Kahler potential is needed for the implementation of an inflationary phase in $\mathcal{R}^{2}$ cosmology. Hence, we will set $W\left(\Phi^{i}\right)=f_{a b}\left(\Phi^{i}\right)=0$. More general theories with $W\left(\Phi^{i}\right) \neq 0$ and $f_{a b}\left(\Phi^{i}\right) \neq 0$ will be discussed elsewhere [8]. We note here, however, that non-zero values for these functions need not, in general, alter the conclusions of this paper. The physical component fields of a chiral matter superfield $\Phi^{i}$ are denoted by $\Phi^{i} \sim\left(A^{i}, \chi^{i}\right)$. The scalar component fields $A^{i}$ are coordinates of a complex Kahler manifold with Kahler metric $g_{i \bar{j}}$ [9, 10]. Under Kahler transformations

$$
K\left(A^{i}, \bar{A}^{i}\right) \rightarrow K\left(A^{i}, \bar{A}^{i}\right)+F\left(A^{i}\right)+\bar{F}\left(\bar{A}^{i}\right)
$$

where $F\left(A^{i}\right)$ is an arbitrary holomorphic function of $A^{i}$. Simultaneously, all matter fermions $\chi^{i}$ rotate as [7, 10]

$$
\chi^{i} \rightarrow e^{\frac{\kappa^{2}}{4}(F-\bar{F})} \chi^{i}
$$

where $\kappa^{2}=8 \pi$. The relevant part of the tree-level component field matter Lagrangian 
is given by

$$
\mathcal{L}=\ldots-i g_{i \bar{j}} \bar{\chi}^{\bar{j}} \bar{\sigma}^{m} \mathcal{D}_{m} \chi^{i}+\ldots
$$

where

$$
\begin{aligned}
g_{i \bar{j}} & =\partial_{i} \partial_{\bar{j}} K \\
\mathcal{D}_{m} \chi^{i} & =\left(\partial_{m}-a_{m}\right) \chi^{i}+\ldots
\end{aligned}
$$

Invariance of this Lagrangian under Kahler transformations is achieved through the appearance of the Abelian Kahler connection $a_{m}$ in the covariant derivative of $\chi^{i}$ [11. This connection is a composite field given by

$$
\begin{aligned}
a_{m}= & \frac{\kappa^{2}}{4}\left(\partial_{i} K \partial_{m} A^{i}-\partial_{\bar{j}} K \partial_{m} \bar{A}^{\bar{j}}\right) \\
& +\frac{i \kappa^{2}}{4} g_{i \bar{j}}\left(\chi^{i} \sigma_{m} \bar{\chi}^{\bar{j}}\right)
\end{aligned}
$$

Under Kahler transformations, it follows from (1) and (2) that $a_{m}$ transforms as an Abelian connection

$$
a_{m}^{\prime}=a_{m}+\frac{\kappa^{2}}{4} \partial_{m}(F-\bar{F})
$$

and, hence, the Lagrangian (3) is invariant. We emphasize that any matter Lagrangian coupled to supergravity, for any Kahler potential $K$, automatically displays Kahler invariance at the tree-level. At the one-loop level, however, Kahler symmetry might be anomalous. In this paper, we are interested in the subclass of triangle graphs where two of the three external legs are space-time spin connections $\omega_{m a}{ }^{b}$. The third external leg is the Kahler connection $a_{m}$, while the matter fermions $\chi^{i}$ run around the loop. This aw $\mathrm{w}$ graph contributes a complicated non-local expression to the one-loop effective action. Its variation under Kahler transformations is, however, readily obtained from the appropriate index-theorem [12]. The result is

$$
\delta_{K} S_{1-\text { loop }}=-\frac{n \kappa^{2}}{768 \pi^{2}} \int d^{4} x \sqrt{g} \mathcal{I} m F\left(A^{i}\right) \mathcal{R}_{m n b}{ }^{a} \tilde{\mathcal{R}}^{m n}{ }_{a}{ }^{b}
$$

where $n$ denotes the number of matter superfields $\Phi^{i}$. We now seek to find the supersymmetric extension of the component expression (7). Since at no stage has supersymmetry been assumed to be broken, the $\mathcal{R} \tilde{\mathcal{R}}$ term in (7) should be contained in the highest component of a superfield. To find this superfield, we will use some of 
the standard techniques employed in deriving component field expressions from superspace structures [13]. The bosonic curvature tensor $\mathcal{R}_{m n b}{ }^{a}$ is related to the curvature superform $\mathrm{R}_{b}{ }^{a}=\frac{1}{2 !} d z^{N} d z^{M} \mathrm{R}_{M N b}{ }^{a}$ by

$$
R_{m n b}{ }^{a} \mid=\mathcal{R}_{m n b}{ }^{a}
$$

where the bar denotes projection to the lowest component of the superfield. We now use the fact that the coefficients $R_{m n b}{ }^{a}$ of the superform $R_{b}{ }^{a}$ are expressable [14 in terms of the covariant supergravity superfields $R, G_{\alpha \dot{\alpha}}, W_{\alpha \beta \gamma}$ and their hermitian conjugates. Then, using the decomposition properties of the superform $R_{b}{ }^{a} R_{a}{ }^{b}$ [13], one readily finds that

$$
\mathcal{R}_{m n b}{ }^{a} \tilde{\mathcal{R}}^{m n}{ }_{a}^{b}=\frac{i}{16}\left(\mathcal{D}^{2}-8 R^{\dagger}\right) T \mid+ \text { h.c. }+\ldots
$$

where

$$
T=\left(\overline{\mathcal{D}}^{2}-8 R\right)\left(16 R^{\dagger} R-\frac{5}{2} G_{\alpha \dot{\alpha}} G^{\alpha \dot{\alpha}}\right)+32 W^{\alpha \beta \gamma} W_{\alpha \beta \gamma}
$$

and where the deleted terms contain the gravitino and auxiliary supergravity fields. It then follows that the superfield expression for the anomaly containing (7) is given by

$$
\delta_{K} S_{1-\text { loop }}=\frac{n}{4} \frac{\kappa^{2}}{768 \pi^{2}} \int d^{4} x d^{2} \Theta \mathcal{E} F\left(\Phi^{i}\right) T+\text { h.c. }
$$

where $\mathcal{E}$ is the chiral density superfield. This equation can be readily integrated and yields, to the lowest non-trivial order in supergravity fields, the effective one-loop Lagrangian density

$$
\mathcal{L}_{1-\text { loop }}=\frac{n}{64} \frac{\kappa^{2}}{768 \pi^{2}} \int d^{2} \Theta \mathcal{E} \bar{D}^{2}\left[T \frac{1}{\square} D^{2} K\left(\Phi^{i}, \Phi^{i \dagger}\right)\right]+\text { h.c. }
$$

Note that for on-shell gravitational superfields, $R=G_{\alpha \dot{\alpha}}=0$, and hence expression (12) reduces to the on-shell result given in [5]. For a constant classical background $\left\langle\Phi^{i}\right\rangle$, this Lagrangian density becomes

$$
\mathcal{L}_{1-\text { loop }}=\frac{n}{4} \frac{\kappa^{2}}{768 \pi^{2}} K\left(\left\langle\Phi^{i}\right\rangle,\left\langle\Phi^{i \dagger}\right\rangle\right) \int d^{2} \Theta \mathcal{E} T+\text { h.c. }
$$

We proceed to expand (13) out in components. Using the results in [15, we arrive at

$$
\begin{aligned}
\mathcal{L}_{1-\text { loop }}= & \frac{n}{4} \frac{\kappa^{2}}{768 \pi^{2}} K\left(\left\langle A^{i}\right\rangle,\left\langle\bar{A}^{i}\right\rangle\right)\left\{-2 \mathcal{R}^{2}+\right. \\
& \left.+5\left(\mathcal{R}_{m n}\right)^{2}+4\left(\mathcal{C}_{\text {mnpq }}\right)^{2}\right\}+\ldots
\end{aligned}
$$


where $\mathcal{C}_{m n p q}$ denotes the Weyl-tensor and the deleted terms contain the gravitino and auxiliary fields. Since we have assumed that the $\left\langle A^{i}\right\rangle$ are all constant, one can use the Gauss-Bonnet theorem

$$
\int d^{4} x \sqrt{g}\left\{\frac{2}{3} R^{2}-2\left(R_{m n}\right)^{2}+\left(C_{m n p q}\right)^{2}\right\}=32 \pi^{2} \chi
$$

where $\chi$ is the Euler number of the space-time manifold, to rewrite expression (14) as

$$
\begin{aligned}
S_{1-\text { loop }}= & \frac{n}{4} \frac{\kappa^{2}}{768 \pi^{2}}\left[\int d^{4} x \sqrt{g} K\left(\left\langle A^{i}\right\rangle,\left\langle\bar{A}^{i}\right\rangle\right)\left\{-\frac{1}{3} \mathcal{R}^{2}+\frac{13}{2}\left(C_{m n p q}\right)^{2}\right\}\right. \\
& \left.-80 \pi^{2} K\left(\left\langle A^{i}\right\rangle,\left\langle\bar{A}^{i}\right\rangle\right) \chi\right]+\ldots
\end{aligned}
$$

We will drop the $\chi$ term, since it is a topological number. We will also drop the term proportional to the square of the Weyl tensor $C_{m n p q}$ since it vanishes for a RobertsonWalker metric, which is the type of space-time metric we consider in this paper. This one-loop action, when added to the gravitational tree-level action, yields the following effective gravitational Lagrangian density for conformally flat space-time metrics

$$
\mathcal{L}_{\text {eff }}=-\frac{1}{2 \kappa^{2}}\left\{\mathcal{R}+\frac{n}{72} K\left(\left\langle A^{i}\right\rangle,\left\langle\bar{A}^{i}\right\rangle\right) \mathcal{R}^{2}\right\}
$$

We have dropped the terms containing the auxiliary gravitational fields, since they will not contribute any $\mathcal{R}^{2}$-terms to (17). We emphasize that expression (17) is only valid for constant $\left\langle A^{i}\right\rangle$. It will be shown below that this assumption is justified for the initial conditions relevant to inflation. The effective Lagrangian (17) is, obviously, not invariant under Kahler transformations (1), since it includes the anomalous diagram $a \omega \omega$. A priori, there is nothing wrong with a one-loop effective action which is not invariant under Kahler transformations. In fact, it provides a natural way of generating an $\mathcal{R}^{2}$-term with a field dependent coefficient which potentially can take any desirable value, small or large. We point out that there is yet another anomalous triangle graph capable of yielding such an $\mathcal{R}^{2}$-term, namely the triangle graph $\Gamma \omega \omega$ where $\Gamma^{i}{ }_{j k}$ is the Christoffel connection of the complex scalar manifold with metric $g_{i j}$. The graph $\Gamma \omega \omega$ is anomalous under $\sigma$-model coordinate transformations of the scalar fields $A^{i}$. It contributes a term to the effective action which is of the type (17) with $K$ replaced by $-2 \ln \operatorname{det} g_{i \bar{j}}$ [5, 6]. We will, however, ignore this contribution, since it is negligible compared to (17) for the choice of Kahler potential we use in this paper. We close this 
part of the discussion by writing down the field equations for the effective gravitational Lagrangian (17) coupled to matter fields. They are

$$
\begin{array}{r}
\left(1+\frac{n}{36} K \mathcal{R}\right)\left(\mathcal{R}_{m n}-\frac{1}{2} g_{m n} \mathcal{R}\right)+\frac{n}{4(36)} K \mathcal{R}^{2} g_{m n}+ \\
\frac{n}{36} K\left(\mathcal{D}_{s} \mathcal{D}_{t} \mathcal{R}\right)\left(g_{m n} g^{s t}-\eta_{m}{ }^{s} \eta_{n}{ }^{t}\right)=-\kappa^{2} T_{m n}
\end{array}
$$

We now discuss the classical evolution of a small isotropic and homogeneous region of the universe, as determined by these field equations. The particular evolution we seek is divided into three phases [2]. (i) The first phase is an inflationary phase of superluminal expansion in which the Hubble parameter decays linearly in time with a small slope. (ii) In the second phase, the Hubble parameter approaches a zero value and bounces back. The universe goes into an oscillatory phase in which it is reheated as matter is excited by the oscillating geometry. (iii) Finally, the universe goes over to a radiation dominated Robertson-Walker phase. We begin by considering a small isotropic and homogenous bubble with a Robertson-Walker metric

$$
d s^{2}=-d t^{2}+a^{2}(t)\left[d r^{2}+r^{2}\left(d \theta^{2}+\sin \theta d \phi^{2}\right)\right]
$$

We take this region to be filled with a single chiral superfield $\Phi \sim(A, \chi)$. Furthermore, we choose the Kahler potential for $A$ to be

$$
K(A, \bar{A})=e^{\bar{A} A}-1
$$

Though unusual looking at first, this Kahler potential reduces to the usual WessZumino Kahler potential, $K=\bar{A} A$, for $|A| \ll 1$. Importantly, however, (20) differs dramatically from the Wess-Zumino Kahler potential for $|A|>1$. Although we will use (20) for concreteness on this paper, a large class of simple Kahler potentials, such as $K=\frac{1}{e} \exp \left(e^{\bar{A} A}\right)-1$, would also lead to a satisfactory inflationary cosmology. The gravitational field equations were derived under the assumption that $A$ is a constant, which we now denote as $A_{i}$. In the homogenous bubble, we choose $A_{i}$ to be real and to lie in the range $5.03 \lesssim A_{i} \lesssim 6.04$. The crucial parameter which governs the successful implementation of the inflationary scenario we are about to discuss, is the function multiplying the $\mathcal{R}^{2}$-term in (17), namely $\epsilon_{i}=\frac{1}{72} K$. The above choice for the value $A_{i}$ translates into a value for $\epsilon_{i}$ in the range $10^{11} \lesssim \epsilon_{i} \lesssim 7 \times 10^{15}$. This is precisely 
the range of values for $\epsilon_{i}$ shown [2] to be compatible with observational constraints on scalar and tensorial perturbations (the upper bound) and the requirements of standard baryogenesis and galaxy formation (the lower bound). The non-vacuous field equations are readily obtained from (18) and (19). The $t$ - $t$ component of (18) yields

$$
\dot{\mathcal{R}}=-\frac{1}{2} \frac{H}{\epsilon_{i}}-H \mathcal{R}+\frac{1}{12} \frac{\mathcal{R}^{2}}{H}
$$

where $H=\frac{\dot{a}}{a}$ denotes the Hubble constant. The curvature scalar $\mathcal{R}$ is given by

$$
\mathcal{R}=6 \dot{H}+12 H^{2}
$$

Combining this equation and (21) yields

$$
\ddot{H}-\frac{1}{2} \frac{\dot{H}}{H}+3 H \dot{H}+\frac{1}{12} \frac{H}{\epsilon_{i}}=0
$$

Next, we would like to solve (23) for $H$ in all three cosmological phases discussed above. These solutions were first given in [2]. We discuss them briefly here, since the results will be used elsewhere in this paper. We begin with the inflationary phase, defined by

$$
\begin{gathered}
\left|\frac{1}{2} \frac{\dot{H}^{2}}{H}\right| \ll|3 H \dot{H}| \\
|\ddot{H}| \ll|3 H \dot{H}|
\end{gathered}
$$

Then, (23) becomes

$$
3 H \dot{H}+\frac{1}{12} \frac{H}{\epsilon_{i}}=0
$$

which is solved by

$$
H=H_{i}-\frac{1}{36 \epsilon_{i}} t
$$

where we have set the time coordinate origin at $t_{i}=0$. Solution (26) describes a long phase in which $H$ decreases linearly in time with a small slope. It satisfies condition (24b). At the beginning of the inflationary phase, $t_{i}=0$, condition (24a) becomes $\frac{1}{6 \sqrt{6 \epsilon_{i}}} \ll H_{i}$ and, hence, imposes a lower bound on $H_{i}$. Furthermore, it is clear from (26) that condition (24a) will eventually be violated for large $t$. One can define the end of this inflationary phase to be the time, $t_{e}$, when $\left|\frac{1}{2} \frac{\dot{H}^{2}}{H}\right|=\frac{1}{10}|3 H \dot{H}|$. It follows from (26) that

$$
t_{e}=36 \epsilon_{i}\left(H_{i}-\frac{1}{6} \sqrt{\frac{5}{3 \epsilon_{i}}}\right)
$$


Therefore, the inflationary period is defined by $0 \leq t \leq t_{e}$. In the oscillatory phase, on the other hand, all terms in equation (23) are taken to be of comparable size. Using the ansatz

$$
H=f(t) \cos ^{2} \omega\left(t-t_{0}\right)
$$

where $\omega$ and $t_{0}$ are constants to be specified below, and by demanding that $\left(\frac{\dot{f}}{f}\right)^{2} \ll \frac{1}{6 \epsilon_{i}}$, one finds

$$
f=\left(g_{0}+\frac{3}{8 \omega}\left[2 \omega\left[t-t_{0}\right]+\sin \left(2 \omega\left[t-t_{0}\right]\right)\right]\right)^{-1}
$$

where $\omega=\frac{1}{2 \sqrt{6 \epsilon_{i}}}$ and $g_{0}$ is an arbitrary integration constant. Parameter $t_{0}$ will correspond to the beginning of the oscillatory phase if we further assume that $\left.\left(\frac{f}{f}\right)^{2}\right|_{t_{0}}=\frac{1}{(16) 6 \epsilon_{i}}$. It follows from this assumption that $g_{0}=\frac{3}{\omega}$. Other than the fact that $t_{e}<t_{0}$, the value of $t_{0}$ has not yet been specified. In the period defined by $t_{e} \leq t \leq t_{0}$, neither the inflation solution (26) nor the oscillatory solution (29) is strictly valid. However, following [2], we will assume that (26) is valid in this region and matches continuously onto (29) at $t_{0}$. It follows that

$$
t_{0}=36 \epsilon_{i}\left(H_{i}-\frac{1}{6} \sqrt{\frac{1}{6 \epsilon_{i}}}\right)
$$

The oscillatory period continues until $t_{F}=t_{0}+12 \times 10^{3} \frac{\epsilon_{i}^{3 / 2}}{N}$ where $N$ is the total number of matter fields [2], at which time the Robertson-Walker phase begins. Therefore, the oscillatory period is defined by $t_{0} \leq t \leq t_{F}$. The only constant left unspecified thus far is the initial Hubble parameter $H_{i}$. This can be determined by integrating equation (26) from $t_{i}=0$ to $t_{0}$ to yield the total expansion of the universe. The result is

$$
a\left(t_{0}\right)=a_{i} e^{18 \epsilon_{i} H_{i}^{2}-\frac{1}{12}}
$$

To obtain approximately $60 e$-followings of inflation, it follows that

$$
H_{i}=\sqrt{\frac{10}{3 \epsilon_{i}}}
$$

which is larger than the lower bound discussed earlier. It was shown in [2] that the above solutions lead to a satisfactory theory of inflationary cosmology. However, in our theory, it was necessary to assume that $A$ is a spacetime constant in order to obtain 
these results. Is this assumption consistent? To explore this, let us take $A$ to be a real function of time but independent of the spatial coordinates. Then one can write $A=A_{i}+A^{\prime}(t)$ where $A_{i}$ is the parameter introduced above. Furthermore, we assume that

$$
\left|\frac{A^{\prime}}{A_{i}}\right| \ll \frac{1}{2 A_{i}^{2}}
$$

Under these conditions, the leading order equation of motion for $A$ is relatively simple and found to be

$$
\ddot{A}^{\prime}+3 H \dot{A}^{\prime}=-\frac{1}{72}\left(\frac{A_{i}}{1+A_{i}^{2}}\right)\left[3 \mathcal{R}^{2}-90\left(\dot{H}^{2}+3 \dot{H} H^{2}+3 H^{4}\right)\right]
$$

where $\mathcal{R}$ is given in (22). Note that with the range of values for $A_{i}$ discussed above, constant $A=A_{i}$ is only a solution of (34) if $\mathcal{R}=H=0$, which they are not. It is necessary, therefore, to go back to the original action (12) and to derive the full Einstein equations allowing $A$ to be a function of time. We continue to assume that constraint equation (33) holds. The result is a generalization of (18) which we need not present here. Suffice it to say that (18) is a very good leading order approximation to the Einstein equations as long as

$$
\begin{gathered}
\left|\dot{A}^{\prime} A_{i}\right| \ll \frac{1}{6}\left|\frac{\dot{\mathcal{R}}}{\mathcal{R}}\right| \\
\left|\left(1+A_{i}^{2}\right) \dot{A}^{\prime 2}\right| \ll \frac{3}{8 \pi} H^{2}
\end{gathered}
$$

Therefore, our theory will closely approximate the constant $A=A_{i}$ results, if the solutions of (23) satisfy (33), (35a) and (35b) for all $t \geq 0$. Let us assume that (33), (35a) and (35b) are satisfied. Then equations (35) can be checked for consistency using the solutions for $H$ given above in the inflationary and oscillatory periods. In the inflationary period, where $0 \leq t \leq t_{0}, H$ is given by (26). If we further demand that $\left|\ddot{A}^{\prime}\right| \ll\left|3 H \dot{A}^{\prime}\right|$, then (34) simplifies to

$$
\dot{A}^{\prime}=-\frac{1}{12}\left(\frac{A_{i}}{1+A_{i}^{2}}\right)\left(\frac{\dot{H}^{2}+9 \dot{H} H^{2}+9 H^{4}}{H}\right)
$$

At $t_{i}=0$, it follows from (26) and (32) that

$$
\dot{A}_{i}^{\prime}=-5 \sqrt{\frac{5}{6}}\left(\frac{A_{i}}{1+A_{i}^{2}}\right) \epsilon_{i}^{-3 / 2}
$$


Furthermore, using this result, (22), (26) and (32), equations (35a) and (35b) become $\epsilon_{i} \gg 10^{3}$ and $\epsilon_{i} \gg 10$ respectively, which are indeed satisfied for $\epsilon_{i}$ in the range $10^{11} \lesssim \epsilon_{i} \lesssim 7 \times 10^{15}$. Note that the condition $\left|\ddot{A}^{\prime}\right| \ll\left|3 H \dot{A}^{\prime}\right|$ becomes $\frac{1}{120} \ll 1$ and, hence, is also satisfied. Equation (33) is automatically satisfied since $A^{\prime}$ vanishes at $t_{i}=0$. It is tedious, but straightforward, to show that (33), (35a) and (35b) remain valid everywhere in the inflationary, oscillatory and Robinson-Walker periods. We conclude that our theory will closely approximate the constant $A=A_{i}$ results for $\epsilon_{i}$ in the cosmologically interesting range $10^{11} \lesssim \epsilon_{i} \lesssim 7 \times 10^{15}$.

As discussed earlier, $\epsilon^{(n)} \mathcal{R}^{n}$ terms where $n \geq 3$ tend to destabilize the $\mathcal{R}^{2}$ cosmological scenario if the $\epsilon^{(n)}$ coefficients are sufficiently large. In this paper, all such terms arise through radiative corrections. Recall that our $\epsilon \mathcal{R}^{2}$ term was generated by a Kahler anomalous, three-point one-loop graph involving one Kahler connection and two spin connections. Since Kahler symmetry is violated, and since the anomaly must be non-local, the effective Lagrangian density was found to be of the form

$$
\mathcal{L}_{1-\text { loop }} \propto \kappa^{2} \mathcal{R}^{2} \frac{\square}{\square} K=\kappa^{2} \mathcal{R}^{2} K
$$

for constant $K$. As we showed, $K$ (and, hence, $\epsilon$ ) can be very large. All other $\epsilon^{(n)} \mathcal{R}^{n}$ terms divides into two types; a) those generated by graphs involving at least one external Kahler connection and b) those generated by graphs all of whose external legs are spin connections. First consider type a). Since these graphs do not violate Kahler invariance, the Kahler connection can only appear as the Kahler invariant field strength $F_{K m n}=\partial_{m} a_{n}-\partial_{n} a_{m}$. Furthermore, these graphs must be local. It follows that a graph with $A$ Kahler connection legs and $B$ spin connection legs generates an effective Lagrangian density of the form

$$
\mathcal{L}_{1-\text { loop }}^{\prime} \propto\left(\kappa^{2}\right)^{A+B-2} \mathcal{R}^{B} F_{K}^{A}
$$

For constant $K, F_{K}=0$ and, hence, $\mathcal{L}_{1-\text { loop }}^{\prime}$ vanishes. Now consider type b). These graphs generate an effective Lagrangian density of the form

$$
\mathcal{L}_{1-\text { loop }}^{\prime \prime} \propto\left(\kappa^{2}\right)^{B-2} \mathcal{R}^{B}
$$

These graphs do not, in general, vanish. However, the coefficients, not being field dependent, are always small numbers of order unity. Thus, since the relevant momenta 
are always smaller than the Planck scale, these terms are much too small to distabilize the cosmology generated by the $\epsilon \mathcal{R}^{2}$ term.

We close this paper with a brief, but important, discussion of the input parameters in our theory. As is well known, most theories of inflationary cosmology suffer from the necessity to introduce and fine-tune many input parameters. Generically, most theories, including ours, have five parameters which describe conditions at the beginning of inflation. In the notation of this paper, these are $a_{i}, H_{i}, \mathcal{R}_{i}, A_{i}$ and $\dot{A}_{i}$. In our case, $a_{i}$ can have any value. Furthermore, assuming (24) and (35), which can be thought of as very mild tunings of order $10^{-1}$ or $10^{-2}, \mathcal{R}_{i}$ and $\dot{A}_{i}$ can be expressed in terms of $H_{i}$ and $A_{i}$ respectively. Therefore, in our theory, the only initial condition parameters are $H_{i}$ and $A_{i}$. In order to have at least $60 e$-foldings of inflation, it follows from (32) that $H_{i} \lesssim 10^{-5}-10^{-8}$, depending on the value of $\epsilon_{i}$. This is indeed a fine-tuning, but it is considerably better than the value of $H_{i} \lesssim 10^{-10}$ required in many other inflationary theories. Finally, the range $5.03 \lessgtr A_{i} \lesssim 6.04$ is of the order of the Planck mass and, therefore, is not really a tuning at all. Our theory shares this nice feature in common with chaotic inflation scenarios [16], but is a vast improvement over most other theories of inflation that must specify $A_{i}$ to a high degree of accuracy. Hence, our initial condition parameters involve only one, relatively mild, fine-tuning. Furthermore, most other theories of inflation must introduce a complicated scalar potential energy with many new, highly fine-tuned parameters. In our theory, the input potential for $|A| \ll 1$ is almost irrelevant. We have chosen it to be zero in this paper, but it can be non-vanishing as long as it is naturally supressed for $|A|>1$. We believe, therefore, that our claims of naturalness in the introduction are justified.

We would like to thank P. Steinhardt for pointing out to us the relevance, and possible destabilizing effects, of the higher $\mathcal{R}^{n}$ terms, and for other interesting conversations.

\section{References}

[1] A. A. Starobinsky, Phys. Lett. 91B (1980) 99; L. A. Kofman, A. D. Linde and A. A. Starobinsky, Phys. Lett. B157 (1985) 361.

[2] M. B. Mijić, M. S. Morris and W. M., Suen, Phys. Rev. D34 (1986) 2934. 
[3] K. Maeda, Phys. Rev. D37 (1988) 858; J. D. Barrow and S. Cotsakis, Phys. Lett. B214 (1988) 515.

[4] A. L. Berkin, K. Maeda, Phys. Lett. 245 (1990) 348.

[5] G. L. Cardoso and B. A. Ovrut, Nucl. Phys. B369 (1992) 351; G. L. Cardoso and B. O. Ovrut, University of Pennsylvania preprint UPR-0481T (1991), published in Proc. of the Conference on Strings and Symmetries 1991, Stony Brook, May 20-25, 1991; Proc. of the International Europhysics Conference on High Energy Physics, Geneva, Switzerland, July 25-August 1, 1991; G. L. Cardoso and B. A. Ovrut, University of Pennsylvania preprint UPR-0502T (1992), accepted for publication in Nucl. Phys. B; G. L. Cardoso and B. A. Ovrut, University of Pennsylvania preprint UPR-0520T (1992), published in the Proc. of the XV International Warsaw Meeting on Elementary Particle Physics, Kasimierz, Poland, May 25-29,1992.

[6] J. Louis, PASCOS 1991 Proceedings; P. Nath ed., World Scientific 1991; J. P. Derendinger, S. Ferrara, C. Kounnas and F. Zwirner, Nucl. Phys. B372 (1992) 145 .

[7] E. Cremmer, S. Ferrara, L. Girardello and A. van Proyen, Phys. Lett. B116 (1982) 231; Nucl. Phys. B212 (1983) 413.

[8] G. L. Cardoso and B. A. Ovrut, to appear.

[9] E. Cremmer, B. Julia, J. Scherk, S. Ferrara, L. Girardello and P. van Nieuwenhuizen, Phys. Lett. B79 (1978) 231; Nucl. Phys. B147 (1979) 105; B. Zumino, Phys. Lett. B87 (1979) 203.

[10] E. Witten and J. Bagger, Phys. Lett. B115 (1982) 202; J. Bagger, Nucl. Phys. B211 (1983) 302.

[11] P. Binétruy, G. Girardi, R. Grimm and M. Müller, Phys. Lett. B189 (1987) 83;

P. Binétruy, G. Girardi and R. Grimm, LAPP preprint TH-275/90.

[12] L. Alvarez-Gaumé and P. Ginsparg, Annals of Phys. 161 (1985) 423. 
[13] G. Girardi and R. Grimm, Phys. Lett. B260 (1991) 365.

[14] M. Müller, Nucl. Phys. B264 (1986) 292.

[15] S. Theisen, Nucl. Phys. B263 (1986) 687.

[16] A. D. Linde, Phys. Lett. B129 (1983) 177. 